\title{
A utilização da modelagem matemática na produção de avalanches em pequena escala
}

\author{
Renan Pereira Santos (iD
}

\author{
Fernando de Carvalho Pires(D)
}

\begin{abstract}
Resumo
O objetivo deste trabalho é proceder a um relato de experiência sobre o experimento Avalanches, desenvolvido em uma turma de $2^{\mathrm{O}}$ ano do ensino médio profissionalizante, como metodologia de ensino e aprendizagem do objeto de conhecimento "Logaritmos". Sabendo das grandes alternativas de que se lançam mão na busca por metodologias que possibilitem potencializar o ensino e aprendizagem de matemática nos espaços escolares e da necessidade de construir processos mais significativos, com estímulos contextualizados e a utilização de materiais concretos, esse trabalho apoia-se na teoria da Modelagem Matemática. Os resultados apontaram para um ganho no aprendizado, com o reforço de conceitos matemáticos, o uso de estratégia de ensino que se aproxima do real e que propiciou um ambiente de engajamento discente e investigação científica.
\end{abstract}

Palavras-chave: Modelagem Matemática; Metodologia de Ensino; Logaritmo.

\begin{abstract}
The objective of this work is to carry out an experience report on the Avalanches experiment, developed in a 2nd year class in high school, as a teaching and learning methodology for the "Logarithms" knowledge object. Knowing the great alternatives that make use of in search of methods that enable the teaching and learning of mathematics in school spaces and the need to create more processes, with contextualized stimuli and the use of concrete materials, this work is supported by the theory of Mathematical Modeling. The results pointed to a gain in learning, with the reinforcement of mathematical concepts, the use of a teaching strategy that is close to the real and that provided an environment for student engagement and scientific investigation.
\end{abstract}

Keywords: Mathematical Modeling; Teaching Methodology; Logarithm.

\section{Introdução}

A experiência de muitos alunos com a matemática tem construído paradigmas e percepções negativas sobre esse componente curricular e, até mesmo, criando a errônea faceta de que seu conhecimento é para um grupo reduzido de sujeitos que, comumente, se sobressaem em avaliações somativas. Essa é uma impressão que deve ser mudada, por meio de estímulos e metodologias de ensino e aprendizagem dos conceitos matemáticos, de forma que os instrumentais da matemática sejam empregados para solucionar questões pertinentes à realidade objetiva. 
Pensando nisso, resolvemos trabalhar com a Modelagem Matemática na abordagem do conteúdo de Logaritmos para estudantes de uma turma do segundo ano do Ensino Médio Técnico em Administração de um colégio estadual da cidade de Caetité - Bahia. Por requerer grande abstração e por ser o último a ser estudado entre as funções, o instrumental de Logaritmos acaba sendo pouco explorado. Diferente do que os alunos pensam, esse conteúdo tem importantes e úteis aplicações em situações e problemas reais que, explorados em sala de aula, podem render engajamento e aprendizado significativo.

A escolha por trabalhar com a Modelagem Matemática está alicerçada em diversos autores da temática (vide bibliografia) e, por através dela, ser possível construir um ambiente educativo que convirja para um processo de ensino e aprendizagem mais dinâmico e produtivo. A partir dessa premissa, selecionamos o experimento Avalanches disponível no site do Projeto Matemática Multimídia do Instituto de Matemática, Estatística e Computação Científica (Imecc) da Universidade Estadual de Campinas (Unicamp) ${ }^{1}$, por se tratar de um experimento dinâmico e para reforçarmos a aprendizagem da definição e propriedades operatórias do logaritmo, e introduzirmos o estudo de construção do gráfico da função logarítmica.

O experimento Avalanches consiste na reprodução do evento de avalanches em pequena escala, utilizando materiais simples, mas que é aplicado fazendo um paralelo com esse fenômeno natural de escala real, abordando probabilidade, análise de dados e até a possibilidade de fazer previsões. Para a aplicação do experimento na aula em questão, foram feitas algumas adaptações do roteiro original disponibilizado pelo Imecc.

Trazemos nesse relato de experiência[2] o passo a passo que foi seguido para trabalhar a Modelagem Matemática na atividade em questão, o desenvolvimento do experimento em sala de aula com os aspectos de ensino e aprendizagem observados e a discussão dos resultados encontrados.

\subsection{Passos para Modelar}

Para que o experimento envolvendo Modelagem ocorresse da melhor forma possível foram seguidos procedimentos para o planejamento da atividade, de acordo com o passo a passo elaborado a partir das colocações de Maturana e Varela (1995, p. 71) e Biembengut (2000). As etapas descritas na sequência são essenciais para que através do experimento/aula seja possível alcançar os objetivos de aprendizagem desejados e, os destacamos porque cremos serem úteis no planejamento dos professores que queiram desenvolver atividades dessa natureza. Os passos para modelar são:

- 1ํ "Fenômeno a ser explicado": É preciso, inicialmente, ter bem definida qual a situação/problema a ser trabalhada, estudar sobre ela e elaborar uma descrição detalhada.

- $2^{\mathrm{O}}$ "Hipótese explicativa": A partir da descrição, será feita uma análise criteriosa do fenômeno, propondo e formulando hipóteses, identificando constantes e variáveis envolvidas, modelando, dessa forma, a situação/problema.

- $3^{\mathrm{o}}$ "Dedução de outros fenômenos": Uma vez modelada, realiza-se a testagem do modelo criado, verificando sua aplicabilidade na situação/problema e, assim, descrever e relacionar outros eventos a partir desse modelo.

- 4ํ "Observações adicionais": A partir dos resultados observados, efetua-se uma avaliação e validação do modelo.

${ }^{1}$ Disponível em https://m3.ime.unicamp.br/recursos/1366. 
Vale ressaltar que o processo de modelagem, bem como esses passos, pode ser utilizado em qualquer disciplina escolar. Além de contribuir para a multidisciplinariedade, sua utilização permite o protagonismo do aluno na construção do seu próprio conhecimento, posto que essa metodologia de ensino e aprendizagem confere à aula um cenário investigativo, de engajamento ativo e de forma a consolidar as competências e habilidades matemáticas, proporcionando assim uma aprendizagem significativa.

\section{Avalanches em pequena escala}

O Experimento Avalanches na forma original desenvolvida pelo Imecc tem como objetivos modelar o fenômeno de avalanches, construir gráficos e linearizar gráficos através de logaritmos. Os conteúdos trabalhados durante o desenvolvimento da atividade, além de logaritmos e suas aplicações, são gráficos e funções. É interessante destacar que tal experimento utiliza-se de materiais simples, como milho de pipoca, feijão e um recipiente qualquer (copo descartável, por exemplo), materiais esses fáceis de encontrar e de valor acessível.

Com algumas adaptações no experimento original, esse foi aplicado com duração de duas aulas (de 50 minutos cada). Os objetivos específicos da aula foram: a) utilizar a definição e as propriedades operatórias do logaritmo no desenvolvimento do experimento e; b) introduzir a construção de gráfico da função logarítmica. A habilidade a ser desenvolvida pelos alunos é saber aplicar logaritmos em situações-problemas da realidade. Os materiais utilizados durante a aula foram: folhas para anotações, calculadora, $1 \mathrm{Kg}$ de feijão, $1 \mathrm{Kg}$ de milho para pipoca e copos descartáveis de $150 \mathrm{~mL}$.

Após explicar a dinâmica da aula naquele dia, a turma foi dividida em três grupos com a mesma quantidade de integrantes (pode ser até mais grupos, para que não sobre aluno ocioso), dois deles receberam os grãos de feijão e o outro os grãos de milho para pipoca, além de todos receberem um copo descartável de $150 \mathrm{~mL}$ cada. Cada grupo preencheu o recipiente com o máximo de grãos possível, de modo que se acomodassem e formassem um morrinho sobre o copo.

A partir dessa situação, os próximos grãos foram colocados cuidadosamente, um de cada vez, em qualquer lugar do morrinho até que começaram a cair. A queda desses grãos em excesso chama-se avalanche. O número de grãos que caía em uma avalanche é chamado de intensidade. Na Figura 1, podemos notar o envolvimento do grupo no desenvolvimento do experimento, constatando o quanto a atividade impulsionou o trabalho coletivo entre os colegas, haja vista que cada um ficou com uma função no desenrolar do exercício.

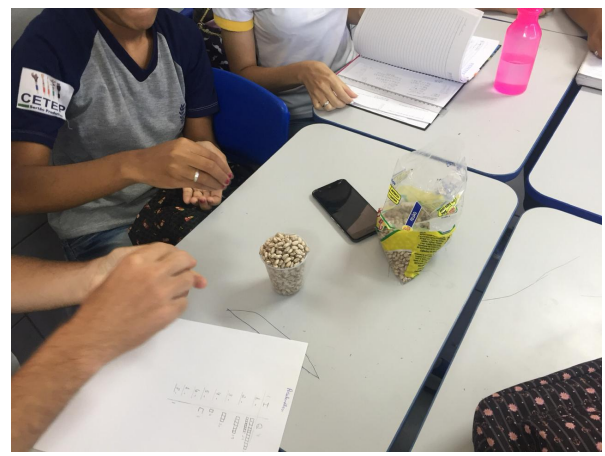

Figura 1: Foto do autor. 
As equipes, portanto, produziram avalanches durante dez minutos, e as intensidades de todas elas foram registradas. Uma tabela foi produzida por cada grupo conforme mostra a Figura 2, onde I é a intensidade, ou seja, a quantidade de grãos que caem de uma avalanche e Q é o número de vezes que a avalanche, de intensidade I ocorreu durante o experimento.

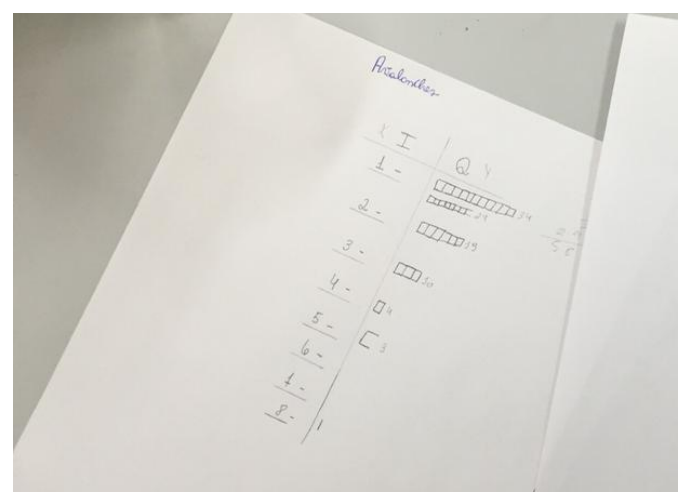

Figura 2: Foto do autor.

Feito isso, os alunos construíram um gráfico de IxQ utilizando os valores reunidos de seu grupo. Os alunos foram questionados se conheciam alguma função que se aproximasse da figura encontrada. Isso suscitou o debate e a observação do que foi encontrado, proporcionando um ambiente investigativo. Como podemos ver abaixo (Figura 3), o formato do gráfico assemelha-se, em linhas gerais, ao formato do gráfico da função $\mathrm{y}=\mathrm{k} / \mathrm{x}$, com $\mathrm{k}>0$.

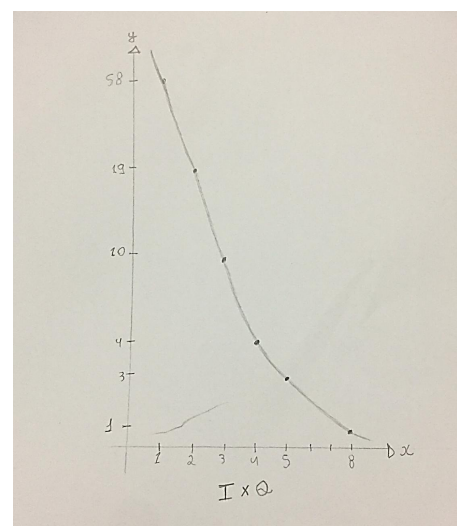

Figura 3: Foto do autor.

Diante disso, encontrou-se um modelo matemático que fosse possível descrever o fenômeno de avalanches, dado pela função:

$$
\mathrm{Q}=\mathrm{a} \frac{1}{\mathrm{I}^{\mathrm{b}}} \Longrightarrow \mathrm{Q}=\mathrm{aI}^{-\mathrm{b}}
$$

onde a e b são constantes. Essa função é adequada para o formato de gráfico do tipo $y=\frac{\mathrm{k}}{\mathrm{x}}$, 
onde as constantes $\mathrm{a}=\mathrm{b}=1$. Restou saber o valor das constantes a e b da equação do gráfico encontrado. Para tal utilizamos logaritmo para transformar a equação dada na equação de uma reta e, posteriormente, encontrar as constantes a partir dos coeficientes facilmente observados no gráfico.

Aplicando logaritmo nos dois lados da equação, obtemos:

$$
\begin{gathered}
\log Q=\log \left(a \frac{1}{I^{b}}\right) \\
\log Q=\log (a)+\log \left(I^{-b}\right) \\
\log Q=\log (a)-b \log (I)
\end{gathered}
$$

Portanto, a equação original foi transformada na equação de uma reta que relaciona os dados coletados no gráfico com seus logaritmos. A fim de facilitar o cálculo para determinar as constantes a e b, a base do logaritmo escolhida foi a base neperiana (e) por fornecer melhores valores para uma posterior produção de gráficos. Logo, substituindo a equação encontrada pelos coeficientes de Q e I representados no gráfico, temos:

$$
\begin{gathered}
\ln 58=\ln \mathrm{a}-\mathrm{b} \ln 1(\mathrm{I}) \\
\ln 19=\ln \mathrm{a}-\mathrm{b} \ln 2(\mathrm{II}) \\
\text { (I) } 4,06=\ln \mathrm{a}-\mathrm{b}(0) \Longrightarrow \ln \mathrm{a}=4,06 \Longrightarrow \mathrm{a}=58 \\
\text { (II) } 2,94=4,06-\mathrm{b} \cdot 0,69 \Longrightarrow \mathrm{b}, 69 \mathrm{~b}=4,06-2,94 \Longrightarrow \mathrm{b}=\frac{1,12}{0,69} \Longrightarrow \mathrm{b}=1,62
\end{gathered}
$$

Com os valores de a e b podemos determinar a equação que se aproxima do gráfico construído a partir dos dados colhidos através do experimento. A equação encontrada, chamando Q de y e I de $\mathrm{x}$, nesse caso foi:

$$
\mathrm{y}=58 \mathrm{x}^{-1,62}
$$

Com o auxílio do aplicativo de geometria dinâmica GeoGebra foi construído o gráfico da equação encontrada (Figura 4) que se aproxima, resguardando as devidas proporções, do gráfico desenhado pela equipe através dos dados coletados do experimento.

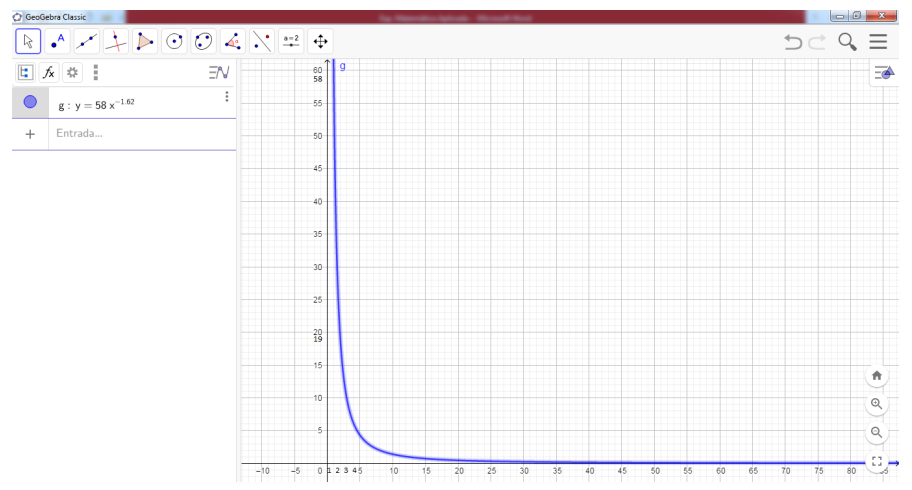

Figura 4: Representação da curva $\mathrm{y}=58 \mathrm{x}^{-1,62}$ no aplicativo GeoGebra Classic. 
Após o desenvolvimento da parte prática e dos cálculos do experimento, os alunos foram conduzidos a reflexões sobre a aplicação do mesmo. Tais reflexões bem como os resultados do trabalho desenvolvido em sala de aula, são abordadas na próxima seção.

\section{Resultados e discussão}

Na parte final do experimento e em diálogo com os alunos, foram suscitadas questões pertinentes relativas à aplicação matemática dos conhecimentos de logaritmo. Usando da análise de dados e da probabilidade, mesmo que de forma implícita para os alunos, eles foram questionados sobre conclusões poderíamos deduzir com relação aos dados da atividade. Observando o gráfico, a maioria dos estudantes chegou à conclusão de que, quanto menor a intensidade, maior era a probabilidade de ocorrerem avalanches, e, quanto maior a intensidade, diminuía a probabilidade de ocorrer o evento.

Tal conclusão a que chegaram os alunos, de fato, pode ser observada no mundo real quando avalanches de terra ou água em menor intensidade ocorrem com frequência nos diversos lugares devido à chuva, por exemplo. As avalanches de maior intensidade, embora façam mais estragos, são mais difíceis de acontecer. Essa conclusão também permite fazer previsões de que eventos de menor intensidade acontecerão com mais frequência que os de maior força, isso pode ser aplicável em outros fenômenos naturais tais como terremotos, temporais etc.

Chegar a essa dedução é importante para desenvolver no aluno a habilidade de interpretar gráficos, previsões e informações probabilísticas tão presentes em noticiários, redes sociais e atividades profissionais.

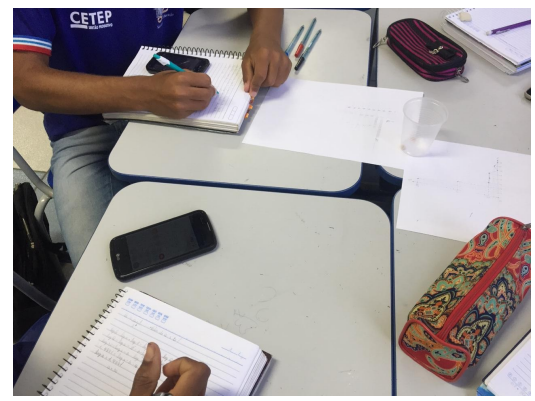

Figura 5: Trabalho em grupo. Foto do autor.

Para os objetivos da aula, o experimento realizado possibilitou reforçar conceitos do conteúdo de logaritmo, com ênfase para as propriedades operatórias e logaritmos de base neperiana. Durante a realização dos cálculos os alunos puderam relembrar as propriedades que envolvem a multiplicação e divisão na função logarítmica, artifícios e caminhos que as propriedades oferecem e que ajudam a solucionar o problema que está posto. Também os alunos passaram a conhecer o logaritmo neperiano que é, digamos, mais fácil de se calcular em calculadoras ou encontrar em tabelas.

Nesse sentido, o experimento também serviu para fixar o que já havia sido estudado até então (função e propriedades do logaritmo) e introduzir a sequência do conteúdo (gráfico e logaritmo neperiano), e o interessante é que foi através de uma atividade prazerosa, interacionista e investigativa, o que vai ao encontro de tendências educacionais em alta na atualidade, como a educação 
"mão na massa" que consiste, basicamente, no aprendizado que passa pelas mãos e centrado no aluno. Além do mais, a utilização de materiais concretos facilitou o engajamento, a imaginação e a observação, tão importantes para o aprendizado.

Durante o desenvolvimento do experimento, foi observado como o aluno tornou-se peça ativa e crítica[4] no processo de aprendizagem, construindo conceitos e aprimorando habilidades. Esse envolvimento característico de quando se usam experimentos em sala de aula foi observado nessa atividade, que foi importante também para sair daquela automaticidade dos exercícios de lista e do livro didático. Por meio da atividade, ainda foi incentivado o trabalho em grupo, e os alunos de forma coletiva buscaram resolver o problema proposto, modelando e utilizando os conhecimentos já adquiridos que eles achavam não servir para nada.

Quanto à avaliação, ela foi feita no decorrer de todo o processo, observando o envolvimento de cada estudante na atividade e acompanhando quais seriam as dificuldades de cada grupo. As dúvidas foram esclarecidas no grupo quando se restringia a apenas um, ou na lousa quando se tratava de uma dificuldade comum entre mais de um grupo.

Por fim, essa atividade que utilizou da modelagem em seu desenvolvimento estimulou a criatividade na formulação e resolução de problemas e incentivou a investigação científica. De acordo com isso, Bassanezi (2002, p. 17) afirma que esse tipo de atividade "é também um método científico que ajuda a preparar o indivíduo para assumir seu papel de cidadão". Logo, podemos notar a importância de aprender as aplicações de conceitos matemáticos que servem para a vida.

\section{Considerações finais}

Diante do que foi exposto, ressaltamos a importância de o professor de matemática reinventarse e realizar atividades lúdicas e experimentais na sala de aula, bem como a importância da Modelagem Matemática como um caminho para a melhoria do ensino e aprendizagem desse campo do conhecimento. Não precisa de muito, apenas de criatividade, para mostrar aos nossos alunos que é possível interpretar e ler o mundo que nos rodeia através de instrumentais da matemática.

Destacamos que se aprende mais e melhor quando o conteúdo pode ser entendido como algo tangível e aplicável em nosso dia a dia ou naquilo que nos circunda. Nesse sentido, a experimentação permite a manipulação laboratorial, obtenção de dados, testagem e validação de um modelo matemático em um processo dinâmico, produtivo, de teorização e consolidação de conteúdos.

Embora sem trazer receitas mágicas, expomos uma estratégia de ensino que pode ser eficiente em sala de aula, visto sua possibilidade de ler, interpretar, observar, argumentar e refutar informações cotidianas, através do conhecimento matemático. Mesmo tratando-se de conceitos matemáticos de grande abstração (logaritmo), identificamos ser possível abordá-la com outras metodologias de ensino que se insurgem como alternativas aos formatos historicamente empregados por alguns professores.

\section{Referências}

[1] Bassanezi, R. Ensino - aprendizagem com Modelagem matemática. $3^{\mathrm{a}}$ ed. Editora Contexto: São Paulo, 2002. Disponível em: <https://www.researchgate.net/publication/256007243_Ensino_-aprendizagem_com_Modelagem_matematica>. Acesso em: 11/01/2021.

[2] Bicudo, Maria Aparecida Viggiani. "Pesquisa em Educação Matemática". Revista Pro-Posições. Vol. 4, no 1 [10], p. 18-23, março de 1993. 
[3] Biembengut, M. S. Modelagem \& Etnomatemática: pontos (in)comuns. I Congresso Nacional de Etnomatemática, 1, São Paulo, 2000. Anais. Disponível em: <http://www2.fe.usp.br/ etnomat/ site-antigo/anais/MariaSalettBiembengut.html>. Acesso em: 11/01/2021.

[4] Freire, P. Pedagogia da Autonomia: Saberes Necessários à Prática Educativa. São Paulo: Paz e Terra, 1996.

[5] Malheiros, A. P. dos S. A produção matemática dos alunos em um ambiente de modelagem. Dissertação de Mestrado. Universidade Estadual Paulista: Rio Claro, 2004. Disponível em: <http://www.rc.unesp.br/gpimem/downloads/dissertacoes/malheiros_aps_me_rcla.pdf >. Acesso em: 11/01/2021.

[6] Maturana, H. R. e Varela, F. G. A Árvore do Conhecimento, tradução de Jonas Pereira dos Santos. Editora Psy II: Campinas, 1995.

[7] Skovsmose, O. Educação Crítica - Incerteza, Matemática, Responsabilidade. São Paulo: Cortez, 2007.

Renan Pereira Santos Secretaria da Educação do Estado da Bahia $<$ renan.psantos96@gmail.com>

Fernando de Carvalho Pires Universidade Estadual Paulista - UNESP $<$ fernando.carvalho3108@gmail.com>

Recebido: $14 / 01 / 2021$ Publicado: 03/11/2021 